\title{
Les jardins zoologiques et la ville : Quelle nature pour le Biodôme de Montréal ?
}

\section{Zoological gardens and the city: What is Nature for Montreal's Biodôme?}

ATER, Ecole Normale Supérieure, Paris

\section{Résumé :}

Le Biodôme de Montréal est un modèle des nouveaux types de jardins zoologiques qui ont émergé à partir des années 1980 et qui transcrivent un rapport renouvelé des sociétés occidentales, très largement urbanisées, à la nature. A travers l'analyse de cette institution ce sont les rapports entre l'homme et l'animal et plus largement la définition de la nature qui sont questionnés, laissant transparaître, au premier abord, un affaiblissement de l'opposition trop nette entre les catégories de nature et de culture. Cependant, plus profondément, il apparaît que les citadins cherchent surtout à se confronter à une nature exotique et sauvage, tout en restant loin des dangers et de la fatigue qu'imposent un vrai voyage. C'est donc une nature édulcorée qui est mise en scène et dont l'altérité même reste étroitement confinée dans un espace précis afin de maintenir valide la distinction entre l'homme et la nature.

\begin{abstract}
:
Montreal's Biodôme can be used as a model of the new type of zoos that emerged in the 1980s. They reflect the changing relationships between Westerner urbanized societies and Nature. Relationships between Men and Animals and the definition of nature are questioned through the analysis of the Biodôme: first, the opposition between Nature and Culture appears to be less and less relevant ; then, the city dwellers appear to be only seeking for direct confrontation with an exotic and wild nature, without any of the hazards or tiredness that supposes a real travel. It is a sweetened Nature that is showed and its "otherness" is locked in a specific place in the city, so that Mankind and Nature remain as two opposite concepts.
\end{abstract}

\section{Mots-clés :}

Biodôme, nature, jardin zoologique, animal, homme, altérité, exotisme, ville.

\section{Key-words:}

Biodôme, Nature, zoological garden, animal, Mankind, otherness, exoticism, city.

\section{Introduction}

Il est désormais acquis que la nature n'est pas une réalité prédéfinie mais bien un concept qui se construit en fonction des époques et des sociétés (Anderson, 2000 ; Latour, 1991, 2001; Moscovici, 1968; Williams, 1997) : la distinction homme/nature, si fondamentale dans la culture occidentale, n'existe pas en tant que telle dans la plupart des autres modèles de société (Fait H. A. in Terrain, 2000 ; Willis, 1990). Il s'agit de construire un concept qui assure alors la répartition et les relations entre l'humain et le non humain. 
Chaque société décide ainsi en permanence de ce qui est naturel et de ce qui ne l'est pas. La civilisation occidentale a présenté comme universelle, car véridique, sa définition de l'homme en tant qu'être supérieur, puisque dégagé de la nature. De ce fait, c'est toute la construction sociale du concept qui s'est trouvée masquée. La ville se présente alors comme l'aboutissement d'un processus de civilisation, qui la place à l'antipode absolu de la nature. Pourtant, il se trouve tout de même deux lieux permanents et de taille importante au cœur de la ville où elle est présentée : le jardin public et le jardin zoologique. Il semble même que ces installations soient associées à la ville dès les origines, comme en témoignent les jardins de Babylone et les premières ménageries qu'on y trouvait (Kisling V.N., 2001), ces aménagements manifestant eux aussi l'urbanité, au point qu'aujourd'hui quasiment toutes les grandes villes possèdent un parc zoologique et, au moins en Europe, plus aucun projet urbain important ne saurait se passer d'un volet espace vert. Ainsi, s'intéresser au jardin zoologique en particulier, c'est lire toute l'ambiguïté des rapports entre les citadins, la nature et leur ville : pourquoi et comment présente-t-on ce qui est sauvage ?

Une première réponse peut venir de ce que la mise en scène de la nature a longtemps semblé répondre principalement à une logique de domination et de maîtrise. Au XVII ${ }^{\mathrm{e}}$ et $\mathrm{XVIII}^{\mathrm{e}}$ siècle, les ménageries, comme celles de Versailles ou de Schönbrun (Batary É et Hadouin-Fugier É, 1998) sont particulièrement liées au pouvoir : il s'agit de regrouper dans le château d'un prince ou d'un roi le plus d'espèces possibles, placées dans de petites cages accolées les unes aux autres. Le but de ces installations est de célébrer la gloire du roi, sa richesse (puisque se procurer des espèces exotiques est alors extrêmement cher) et son pouvoir sur l'univers, puisqu'il est capable de donner son ordre à la nature en maîtrisant les bêtes les plus grosses et les plus dangereuses, comme les lions, les éléphants ou les rhinocéros. Le jardin relève de la même logique de domination de la nature puisque l'homme plie les plantes à sa volonté, sa puissance et à l'ordre rationnel qu'il veut lui imposer que ce soit celui de la symétrie et de la juxtaposition de plans du jardin à la française, dont le modèle reste Versailles, des courbes du jardin à l'anglaise ou de la réorganisation des différents éléments qui composent le monde dans les jardins de style japonais. De même, lorsque le jardin zoologique devient public, après la Révolution française, il constitue lui aussi une entreprise scientifique dans l'esprit de l'encyclopédisme : recenser, classer et étudier l'animal. Cette domination s'inscrit également dans l'espace des jardins botaniques où s'affiche un classement des plantes qui suit un ordre de la science par leur disposition même dans l'espace et des séries de panonceaux portant leur nom. Le zoo comme le jardin public célèbre donc finalement tout autant la maîtrise sur la nature que la ménagerie ou le jardin royal, mais cette fois en rapport avec le pouvoir de la science. Puis, à la fin du $\mathrm{XIX}^{\mathrm{e}}$ et au début du $\mathrm{XX}^{\mathrm{e}}$ siècle, celui-ci devient une vitrine de l'impérialisme pour des pays qui se sont lancés dans la colonisation. Le zoo a alors pour but d'affirmer la domination et la maitrise sur les animaux exotiques et dangereux des colonies, et donc indirectement sur les colonies elles-mêmes, à la fois parce que le système de cages et de fossés indique bien un rapport de force, mais aussi parce que le zoo permet de connaître et donc de s'approprier les animaux de l'empire. L'altérité radicale est en quelque sorte exorcisée par le rapport de domination très fort qui est clairement affirmé.

Cependant, depuis la fin des années 1980, il semble s'être produit une sorte de retournement dans la façon dont la nature est présentée en ville par une nouvelle génération de zoo, comme celui du Bronx avec son espace Jungle World. Ici, l'altérité de la nature, loin d'être réduite par une domination affirmée parait, au contraire, exacerbée par la disparition des cages et la reconstitution d'écosystèmes à l'aide de techniques sophistiquées. Plus que dans le cas du jardin ou du zoo classique, on a là une véritable réintroduction de la nature sauvage pour elle-même dans la ville, ce qui redonne de l'acuité à la question de sa fonction. 
Le Biodôme de Montréal nous servira de fil directeur dans l'analyse de ce retournement des conceptions et des représentations de la nature du fait de son caractère de modèle à plusieurs égards.

Tout d'abord, il s'agit sans doute chronologiquement du premier exemple de ce nouveau type de représentations de la nature en ville, avec une conception datant de la fin des années 1970 (Batary É et Hadouin-Fugier É, 1998). Il faut voir que le Biodôme a été largement imité par d'autres institutions. De fait, le succès public de l'institution tant en terme de fréquentation que de rayonnement international a poussé de nombreux zoos à adopter le même type de présentation que le Biodôme pour au moins une part de leur collections (le zoo du Bronx, de Toronto, de San Diego, entre autre). Depuis sa création, le Biodôme accueille ainsi en moyenne 950000 visiteurs annuels dont près de la moitié ne sont pas canadiens. Les groupes scolaires et touristiques comptent pour $22 \%$ de la fréquentation annuelle. Par ailleurs, on peut noter qu'environ un quart du total des visiteurs à moins de 18 ans (Services du Biodôme, 2003).

Le Biodôme reste également un modèle parce qu'il est encore le seul à proposer les représentations de quatre écosystèmes (la forêt tropicale, la forêt laurentienne, le SaintLaurent marin et la zone polaire) entièrement recréés -tant en ce qui concerne la faune, la flore que le climat- dans le même lieu, ce qui accroît la complexité spatiale de l'ensemble : en quelques minutes le visiteur peut avoir l'illusion de parcourir plusieurs milliers de kilomètres. On assiste donc à un emboîtement vertigineux et inhabituel des échelles pour un lieu, du micro-local de l'espace où le visiteur se situe et observe une cascade, à l'espace des très grands ensembles (Amérique du Nord principalement) qui lui est suggéré à travers le caractère paradigmatique des espaces représentés. Il faut cependant voir qu'en dehors de la forêt tropicale, les paysages mis en scène sont essentiellement canadiens voire québécois et peuvent s'analyser comme de véritables monuments naturels nationaux qui s'inscrivent pleinement dans un processus de construction identitaire.

En dernier lieu, le Biodôme offre la particularité de présenter un dispositif spatial de fermeture de l'ensemble au monde extérieur très net, du fait de l'architecture du bâtiment sous forme de voûte, ce qui souligne son rôle très particulier dans la cité puisqu'il faut le séparer du reste du tissu urbain. Or, ce type de dispositif se retrouve dans l'ensemble des institutions qui présentent la nature en ville mais souvent de manière moins nette. Cependant, nous verrons que bien que de nouvelles conceptions aient réellement déstabilisé la frontière entre l'homme et la nature, au point de remettre en cause la légitimité des jardins zoologiques, fondée sur les loisirs et le divertissement, l'intérêt pour les visiteurs reste de se confronter à une nature sauvage et exotique. La visite doit permettre une véritable interaction avec ce qui est encore perçu comme l'altérité radicale -l'animalité- et par-là même satisfaire un besoin de nature mais aussi réaffirmer sa propre identité, tout en restant préservé des dangers réels. Car, au final, la nature qui nous est présentée n'est libre que de manière illusoire, en conformité avec les attentes du public. Le contrôle sur l'animalité se fait plus discret, qu'on le camoufle derrière des dispositifs spatiaux invisibles ou des mots comme conservation ou protection mais reste bien réel : il s'agit de persuader le public qu'il déambule dans un espace ouvert, tout en le protégeant de toutes les agressions matérielles ou symboliques que peut lui faire encourir sa confrontation avec la nature, notamment en lui garantissant que l'espace dans lequel il se trouve est tout à fait exceptionnel dans la ville (Philo, 1995). Le Biodôme reste un espace fermé dans lequel on entre et on ne sort qu'en passant à travers une série de seuils.

Cette étude a été menée en 2003/2004 à partir de plusieurs sources. Tout d'abord, les archives du Biodôme, où près de deux mille documents sur les projets de construction, de scénographie mais aussi des plans, des prospectus, des dossiers de presse, des publicités et des statistiques, ont étés dépouillés. Il a ainsi été possible d'exploiter les sous-produits d'une enquête interne au Biodôme menée en 1996 (Tarpin Ch., Pitre R., 1996) sur les 
comportements de 508 visiteurs observés à l'aide de caméras puis interrogés, pour 83 d'entre eux par questionnaires. Seconde source de renseignement, l'observation du comportement des visiteurs, des animateurs, des agents de sécurité dans les écosystèmes mais aussi dans la boutique et le hall d'entrée de l'institution. Nous avons ensuite mené des entretiens semidirigés d'une heure environ avec le directeur et membre fondateur de l'équipe qui a conçu le Biodôme, un conseiller scientifique, un animateur et le responsable de la Société des Amis du Biodôme (SABM). Enfin, nous avons mené 46 entretiens semi-dirigés de 30 à 45 minutes chacun avec le public du Biodôme. Ces entretiens étaient organisés autour de 26 questions sur la nature, sa présentation au Biodôme et les paysages mis en scènes puis étaient complétés par un échange plus libre. Ces entretiens ont été menés à la fois avec des individus et des groupes de deux à cinq personnes, choisis aléatoirement à la sortie du dernier écosystème du 29 janvier au 17 février 2004.

\section{De nouveaux rapports à la nature \\ 1.1 Quatre écosystèmes reconstitués}

Le bâtiment du Biodôme était à l'origine un vélodrome, construit à l'occasion des Jeux Olympique de Montréal, en 1976. Il fait partie du complexe olympique installé dans le quartier Hochelaga-Maisonneuve, dans l'est de l'île de Montréal. Le choix de l'implantation de l'ensemble dans cette partie de la ville, assez éloigné du centre de la métropole, tenait à une volonté de redynamiser un quartier proche du port et de profiter d'un terrain libre de plus de 55 hectares, longeant la rue Sherbrooke, entre les rues Pie IX et Viau (voir figure 1).

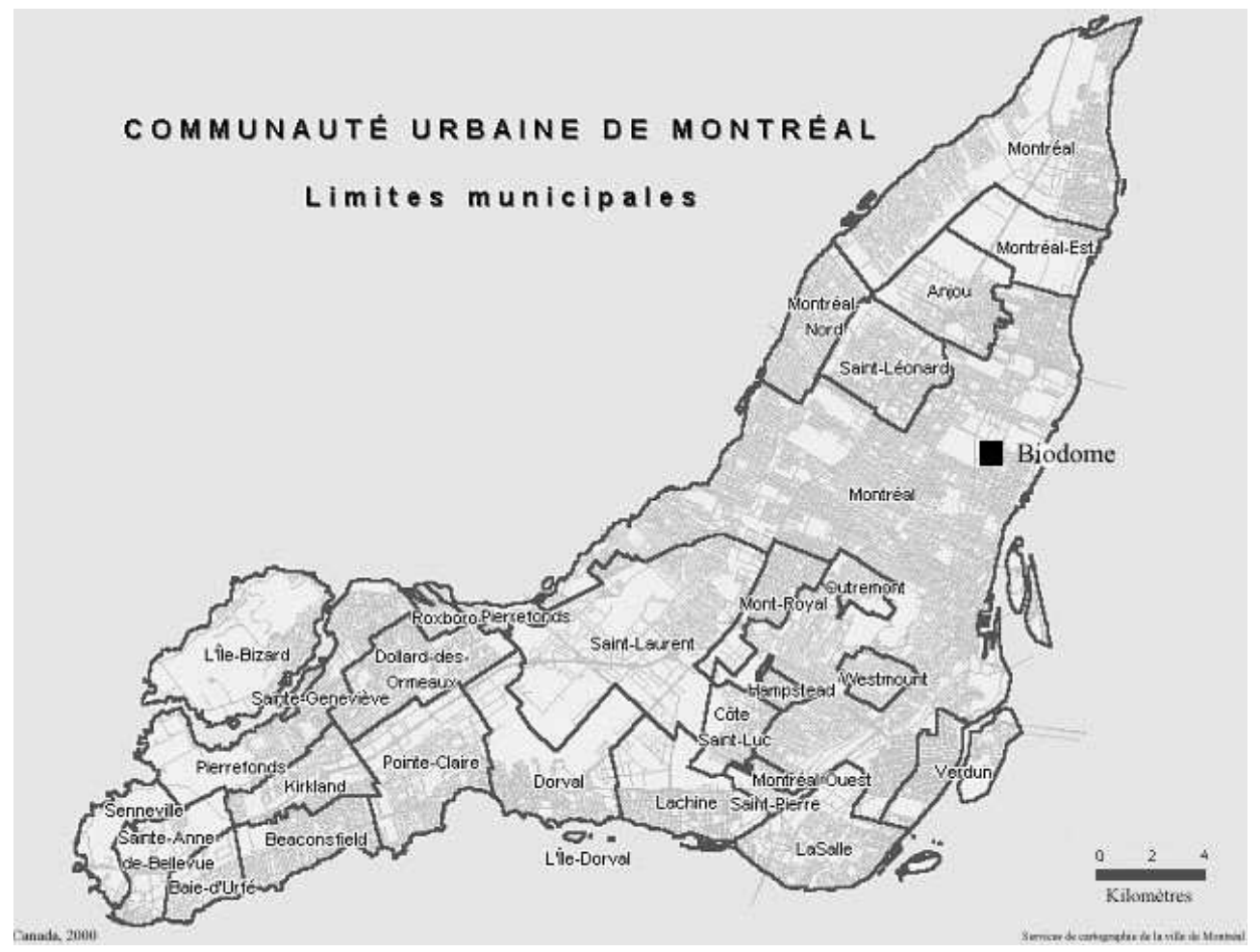

Figure 1 : La C.U.M, 2000, carte des services de la ville de Montréal retouchée par l'auteur

Pendant les années 1980, il apparaît que le vélodrome est constamment sous-utilisé, ce qui entraîne de gros déficits d'exploitation. Or, comme au même moment l'ancien aquarium 
et le jardin zoologique de la ville, totalement dépassés techniquement et dans leur présentation, doivent être rénovés, la ville de Montréal décide de fusionner ces institutions pour créer le Biodôme. Le bâtiment conçu par Roger Taillibert présente quelques avantages majeurs : tout d'abord il se présente comme une sorte de coque en béton précontraint, ce qui limite grandement les déperditions d'énergie et permet également d'avoir une voûte sans appuis intérieurs libérant ainsi une vaste surface à l'intérieur du bâtiment (figure 2). Par ailleurs, la voûte est conçue de telle manière à ce que $60 \%$ de sa surface soit percée par des lanterneaux qui assurent le passage de la lumière extérieure ce qui est essentiel si on espère faire croître des espèces végétales.

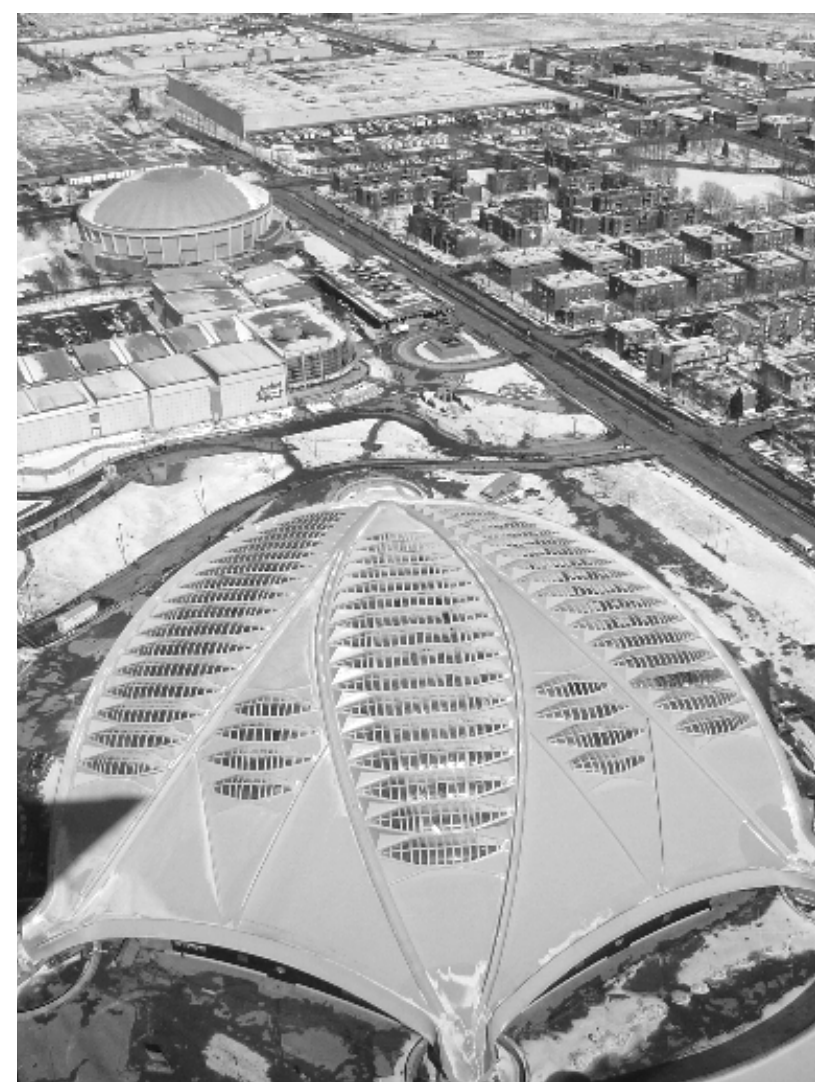

Figure 2 : Le Biodôme vu depuis le mât du stade olympique, 2004, cliché de l'auteur

Une fois que le visiteur a franchi les différents seuils qui lui permettent d'accéder à l'intérieur du Biodôme, il se retrouve au début d'un parcours de 500 mètres qui va le mener à travers les différentes représentations de la nature proposées (figure 1). 


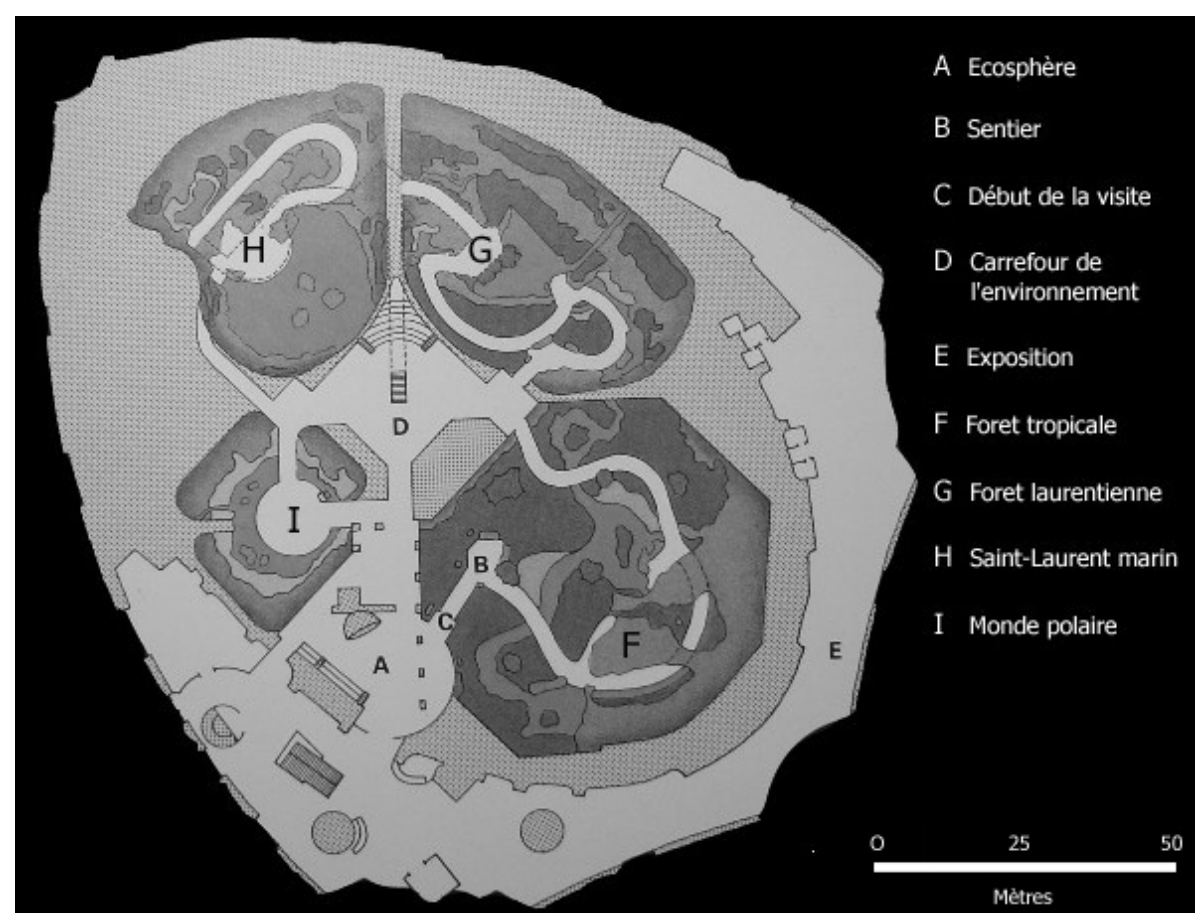

Figure 3 : Plan intérieur du Biodôme, 1993, services du Biodôme, retouché par l'auteur Plan of Montreal's Biodôme's

Tout d'abord, le visiteur traverse la forêt tropicale qui présente un paysage à la végétation très dense et variée. Le sentier passe ainsi devant diverses espèces végétales, des arbres à contreforts aux lianes et autres épiphytes au pied desquels nagent plus de mille poissons, du Piranha commun au Diable rouge. A hauteur du sentier et légèrement en contrebas se trouvent quelques centaines de reptiles et de mammifères. Les strates supérieures de la forêt sont occupées par des oiseaux qui restent relativement peu visibles dans leur majorité (en dehors des espèces de grande taille comme l'Ara) mais qui contribuent à rendre cet écosystème extrêmement sonore. Le paysage reproduit est celui de diverses parcelles de forêt tropicale du Costa Rica.

Un autre sas conduit alors le visiteur dans la forêt laurentienne. Cet écosystème reproduit le paysage qu'on trouve au nord de Montréal, sur le Bouclier canadien. La figure 2 nous permet de voir en bas à gauche de l'image le lac des castors, qui est l'élément central de la représentation, puis, en regardant vers la gauche, un ensemble de peupliers, d'érables et de conifères qui sont installés sur les bordures du lac, ainsi que des escarpements en béton, qui représentent le relief des montagnes laurentiennes. Dans la partie supérieure de l'image, on observe des tubes de ventilation et d'humidification. En entrant, le contraste de température avec la forêt tropicale est saisissant, surtout en hiver, puisque la température (entre 4 et 12 degrés) et l'humidification sont réglés pour produire la dormance ${ }^{1}$ de la forêt qui entraîne notamment la chute des feuilles. L'idée est bien de reproduire les cycles naturels saisonniers.

\footnotetext{
${ }^{1}$ Il s'agit sommairement d'un état d'hibernation végétale.
} 


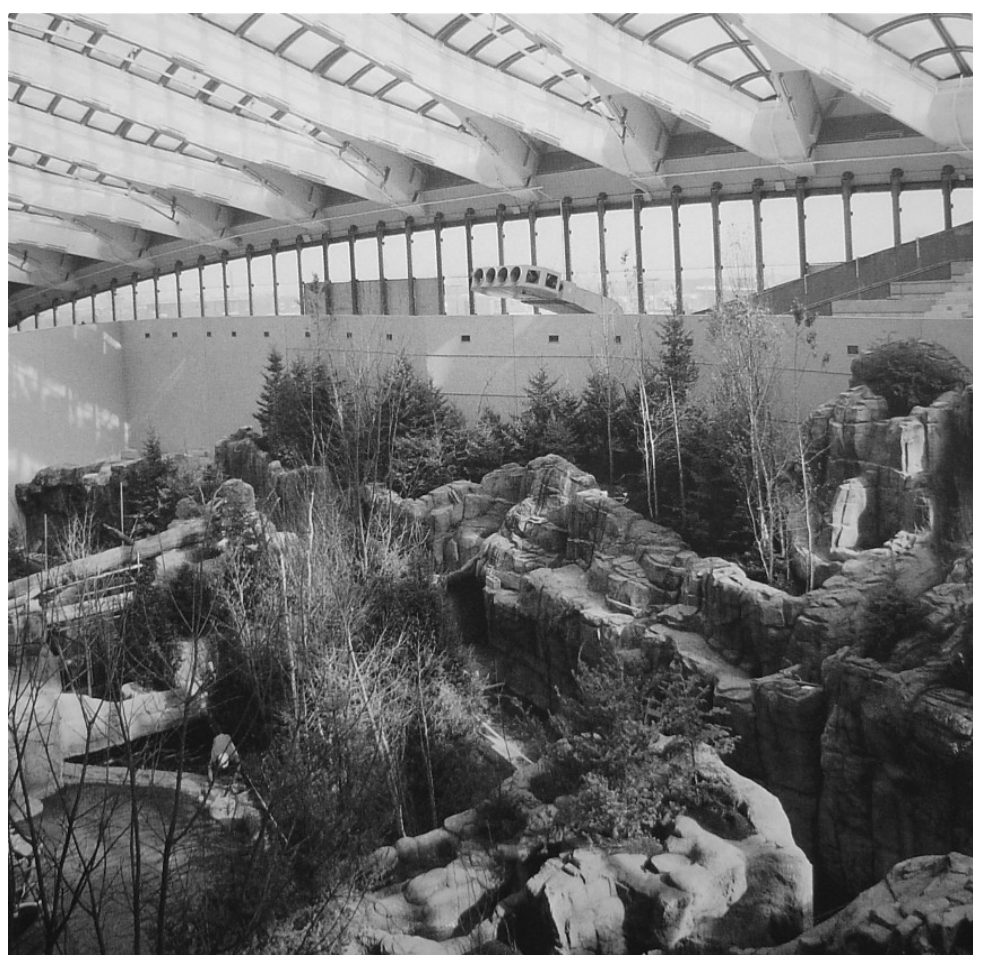

Figure 4 : La forêt laurentienne en préparation, 1992, cliché Pierre Perreault

Making the Laurentien forest

Dans cet environnement vivent, au total, plus de 150 poissons, 30 amphibiens, une vingtaine de reptiles, quatre mammifères et plus d'une centaine d'oiseaux. Le parcours nous dirige ensuite vers le Saint-Laurent marin qui représente le fleuve à partir de l'endroit où ses eaux se mêlent à celles de l'océan. Le visiteur passe tout d'abord devant un vaste aquarium d'eau salée et réfrigérée, qui contient des morues, des bars d'Amérique et autres flétans, puis il ressort dans un paysage minéral qui représente les paysages de l'estuaire. Ce sont de véritables parois rocheuses qui ont été moulées afin de reproduire les escarpements de la rive. L'ambiance marine est assurée par l'odeur d'iode, celle d'algues et par les cris des sternes ou des mouettes qui ne cessent de tourner à l'intérieur de l'écosystème et de se poser sur les différents éléments du décor. Enfin, le parcours se dirige vers l'écosystème du monde polaire. Le milieu est présenté dans une salle relativement petite, avec deux espaces vitrés représentant chacun un des pôles, l'un maintenu à 12 degrés, l'autre à 2 . Il peut alors apercevoir sur des plages de galets et quelques escarpements rocheux des manchots et des gorfous.

Plusieurs points importants dégagent manifestement le Biodôme des représentations habituelles de la nature en ville. En premier lieu, contrairement au jardins et aux zoos classiques, l'objectif affiché n'est pas tant de présenter une nature pliée à la rationalité de l'homme mais bien plutôt une nature vierge de toutes transformations humaines, alors même qu'elle a été tout aussi construite que dans un jardin. Tout l'équipement technique du Biodôme, qui est camouflé autant que possible, doit donner l'illusion que la nature y fonctionne de manière autonome et libre. Par exemple, le visiteur ne voit aucune cage à l'exception de celles du lynx et des animaux du monde polaire. En somme, les animaux semblent évoluer en liberté dans les écosystèmes. L'organisation de la flore selon un modèle qui caractérise les jardins ne se retrouve pas plus puisqu'il s'agit de reproduire un milieu naturel qui préexiste. Enfin, contrairement aux parcs zoologiques classiques, les visiteurs se déplacent à l'intérieur des milieux et y participent, en quelque sorte. Ils sont plongés dans l'atmosphère chaude et humide de la forêt tropicale ou l'ambiance beaucoup plus fraîche et sèche de la forêt laurentienne en hiver. Ils sentent la mer dans le Saint-Laurent marin du fait 
des systèmes de salinisation et de réfrigération de l'eau. Cette immersion totale donne un effet de réalité très profond à l'installation. Ce moyen est alors utilisé par l'institution pour faire ressentir au public qu'il fait partie de la nature.

\subsection{La déstabilisation des frontières homme/animal et de la légitimité des parcs zoologiques}

Ces nouvelles façons de représenter la nature ne sont évidement apparues qu'après un long travail de redéfinition. Si depuis l'antiquité, l'homme occidental n'avait cessé de se penser comme radicalement différent de la nature, un mouvement inverse s'amorce dans la deuxième moitié du XXe siècle. Le changement vient du côté de la confrontation de l'homme avec l'animal. Alors que le propre de l'homme avait souvent été défini par son esprit, c'est-àdire ses capacités cognitives sophistiquées, la découverte que des grands singes ou des perroquets sont capables d'utiliser ces mêmes capacités semble annuler cette spécificité essentielle. Des juristes et des philosophes tentent également de déstabiliser la frontière entre l'homme et l'animal avec parfois des excès notables. Ainsi, en 1975, P. Singer écrit La libération animale, dans laquelle il développe l'idée que les êtres humains exercent sur les autres animaux une tyrannie sans comparaison. Utilisant les outils théoriques du féminisme, il forge le concept de "spécisme» qu'il lie directement au sexisme. Face au "chauvinisme humain », P.Singer propose de mettre en place des «droits des animaux ». Des mouvements comme Greenpeace, People for the Ethical treatment of Animals ou The Animal Liberation Front, dont certains s'inspirent directement des idées de Singer, forment des lobbies qui vont contribuer à obtenir l'adoption de la "déclaration universelle des droits de l'animal » en 1978. C'est notamment à la suite de ces actions que la société civile commence alors à s'intéresser à la protection des espèces en voie de disparition et à la faune « sauvage ». Il apparait alors qu'un tournant « environnementaliste » touche une partie des pays industrialisés avec la mise en place de normes, de lois et de processus de plus en plus stricts de protection de la nature. De ce fait, c'est la possibilité même de l'enfermement de spécimens vivants qui pose problème, non pas seulement de manière théorique, parce qu'elle contredirait un nouvel édifice intellectuel, mais bien parce qu'une nouvelle sensibilité a gagné le public.

Avec la remise en cause de leur légitimité, la plupart des zoos classiques -c'est-à-dire dans lesquels l'enfermement des animaux est manifeste- sont frappés par une image de marque de plus en plus désastreuse. Afin d'échapper à cette crise majeure, le Biodôme fait tout pour essayer de se distinguer de ces institutions en montrant que son projet se fonde sur un concept neuf voire même « unique » : l' " écosystème ». On peut effectivement constater que les milieux ne sont pas conçus pour mettre en valeur les animaux, à la différence des zoos « classiques », mais comme un ensemble d'éléments en interaction possédants la même valeur. Ainsi, il reste quasiment impossible de voir tous les animaux, particulièrement dans la forêt tropicale, puisque de nombreux spécimens peuvent aisément se cacher. La notion d'humanité semble, elle aussi questionnée. Dans le premier espace chargé de présenter la notion d'écosystème, on trouve un panneau clamant: "Les végétaux, source universelle de nourriture, sont indispensables aux animaux et aux humains », alors qu'à côté un documentaire télévisé annonce que «Tous les êtres vivants de la planète (végétaux, animaux -dont l'humain-) sont liés entre eux et à leur environnement physique » (l'italique est de notre initiative). Il est évident que les deux messages sont en pleine contradiction, puisque l'un affirme que l'homme est différent de l'animal, alors que l'autre les place dans la même catégorie. La juxtaposition de ces deux messages traduit peut être la position délicate dans laquelle se trouve le Biodôme, tiraillé entre un discours officiel qui cherche à montrer l'inclusion de l'homme dans la nature et le lourd héritage de toute la pensée occidentale jusqu'à une époque récente, qui garde une influence majeure. 


\subsection{Justifier sa visite : liberté, protection, éducation}

Puisque la principale critique dont souffrent les zoos est le traitement et l'enfermement des animaux, le Biodôme a mis en place un discours officiel assez lissé sur la question. Il s'agit de monter que les animaux ne sont pas que des objets et qu'ils sont donc dignes de respect, en maintenant une densité d'animaux peu élevée et en donnant l'impression qu'ils vivent dans leur «milieu naturel », c'est-à-dire, finalement, qu'ils restent libres. En effet, le Biodôme a eu pour politique d'éliminer autant que possible les clôtures et les contentions visibles. On se rend compte que beaucoup de visiteurs ne se sentent pas gênés de voir des animaux au Biodôme, comme ils le seraient si les cages étaient évidentes. Dans la perception des visiteurs, -ce qui est sans doute le plus important, puisque l'institution existe avant tout pour le public- le Biodôme n'est donc pas un jardin zoologique (les entretiens déterminent que moins d'un visiteur sur six, propose cette définition), mais bien plutôt un lieu où on retrouve une combinaison d'éléments. En somme, l'idée générale est que les animaux vivent mieux en captivité, où ils sont soignés, nourris et protégés des prédateurs, qu'en "vraie » nature. Ainsi, les « animaux sont contents, ils n'ont pas de prédateurs » ou « ils passent un bon moment ». Seul l'espace du lynx ou des manchots suscitent des remarques des visiteurs. Tout d'abord, les contentions (filins métalliques et électriques pour le lynx et cage vitrée pour les manchots), y apparaissent nettement, ce qui rappelle les cages des zoos traditionnels et recrée alors un certain malaise. Cela dit, ces animaux ne sont pas les seuls à être enfermés dans ces conditions: il suffit de s'intéresser au cas des chauves-souris, des grenouilles dendrobates ou de l'anaconda. Cependant, aucun des visiteurs questionnés ne les a mentionnés, ce qui laisse à penser qu'ils établissent une véritable hiérarchie entre les animaux, entre ceux qui semblent suffisamment important pour mériter la liberté et ceux qui laissent indifférents, ceux qui ne semblent pas assez sensibles pour avoir besoin d'un espace de liberté (liberté imaginaire, certes mais qui existe dans l'esprit du public quand il ne voit pas de barrière directe).

Comme l'annonce son site Internet «le Biodôme participe [...] à plusieurs programmes nationaux et internationaux de conservation visant à prévenir la disparition et à favoriser la réintroduction en milieu naturel d'espèces menacées $»^{2}$. De fait, cet aspect de sa « mission» (comme le qualifie lui-même le Biodôme) est un point qui est constamment mis en exergue par l'institution dans ses publications et sur le parcours des écosystèmes mêmes. On y trouve en évidence le logo de l'American Zoo and Aquarium Association (A.Z.A.) qui a accrédité l'institution en tant que parc zoologique respectant certaines normes de qualité. Ainsi, le Biodôme hésite sur la définition de sa propre identité en cherchant à se démarquer des zoos mais, en même temps, en embrasse leur nouveau cheval de bataille : la conservation et la réintroduction des espèces en milieu naturel. Pourtant, l'institution ne possède et ne conserve que sept espèces d'animaux et deux espèces de végétaux menacées sur un total respectif de 250 et 350 espèces. Il faut se rendre compte que ces espèces menacées ne représentent que $2,8 \%$ et $0,6 \%$ du total des espèces possédées. C'est bien qu'il existe un décalage manifeste entre le discours qui met en avant le rôle majeur de la conservation et la réalité des choses, ce en quoi le Biodôme ne diffère pas vraiment des autres jardins zoologiques. De manière générale, le nombre d'espèces en voie de disparition est bien supérieur à la «capacité d'accueil» de tous les zoos de la terre. De plus, sauvegarder des animaux impose des choix qui sont très contestables, montrant qu'il existe une hiérarchie implicite entre les espèces : alors que le zoo choisira avant tout de sauvegarder un singe on peut se demander en quoi la disparition d'un insecte est moins grave en soi. Une des

\footnotetext{
${ }^{2}$ www.ville.montreal.qc.ca/biodome/2-coll/f2_cons.htm, accédé le 3/04/04
} 
meilleures preuves de la puissance du discours diffusé par l'institution est que malgré tout près de $80 \%$ des visiteurs pensent que le Biodôme peut avoir un rôle de sauvegarde des espèces en voie de disparition. Si le Biodôme met en avant avec tant d'empressement ces programmes de conservation et de réintroduction, c'est que ces éléments sont de puissants moyens de justification de l'enfermement des animaux. En montrant que certaines espèces n'existent plus dans la nature ou sont en voie de disparition, on arrive à la conclusion que les zoos sont le seul endroit où ces animaux vont pouvoir survivre, au moins provisoirement, dans de bonnes conditions. Ainsi, le zoo devient une sorte d' "Arche de Noé " (Hancock \& Staszak, 2002) qui protège les animaux en les mettant « hors du monde » temporairement et qui doit permettre d'accroître le nombre de spécimens d'une espèce dans l'espoir de les relâcher dans la nature. Dans cette perspective, les naissances dans l'enceinte du zoo deviennent les preuves de la réussite de l'institution et justifient donc son existence. Ce discours très habile permet ainsi de montrer que les zoos ne cherchent que le bien des animaux et qu'il sont, à leur plus grand regret, obligés de les enfermer à cause de la société (Hancock et Staszak, 2002).

Enfin, pour exprimer sa distanciation vis-à-vis des jardins zoologiques et à l'aspect spectaculaire de divertissement qu'ils présentent, le Biodôme se définit officiellement comme un «musée [possédant] des collections vivantes » (Quatre temps, 1992, p. 30-31). Ainsi, son objectif majeur serait de transformer le rapport du visiteur à la nature, en lui faisant prendre conscience du fait qu'il en fait partie, tout en le poussant à modifier son comportement. Malgré ces objectifs, il apparaît que les visiteurs n'attachent que peu d'importance à l'aspect éducatif de l'institution. Près des trois quarts des visiteurs ne regardent même pas les panneaux, et ce ne sont qu'environ $10 \%$ d'entre eux qui lisent réellement ${ }^{3}$, ce qui signifie bien que le message du Biodôme n'est quasiment pas transmis puisque les panneaux sont supposés être le principal vecteur d'information scientifique. De fait, la rapidité avec laquelle une bonne partie des visiteurs effectuent leur visite indiquent qu'ils ne s'attardent pas pour tenter d'apprendre. A la suite des entretiens il est apparu que la notion d' "écosystème » est, au mieux, mal comprise par le public et, le plus souvent, ignorée. En fait, les animaux sont les vedettes du Biodôme et la végétation est d'avantage perçue comme un décor que comme une composante de l'écosystème. De ce fait, pour le public, le Biodôme reste bien fonctionnellement un zoo et ce malgré tous les efforts de l'institution pour éviter cette assimilation.

Un point fondamental ressurgit donc : qu'y recherchent les visiteurs? Il faut se rendre compte que ce sont essentiellement des citadins qui sont attirés par une nature sauvage et souvent exotique qui n'existe à proximité de chez eux «en vrai», c'est-à-dire comme spécimen vivant, que dans les institutions de type zoologique. C'est la confrontation directe avec ce qui connote l'aventure, la découverte, le courage voire l'authenticité par excellence qui motive essentiellement les visites.

\section{Une nature instrumentalisée}

\subsection{Découverte de l'Ailleurs et complexité de l'espace}

En se fondant sur l'analyse des entrevues, il apparaît que les visiteurs cherchent avant tout à s'immerger dans les écosystèmes pour créer une véritable rupture avec le monde extérieur. C'est d'ailleurs ce critère de vraisemblance qui est l'élément d'explication le plus

\footnotetext{
3 étude interne menée au Biodôme en 1996 par Ch. Tarpin et R. Pitre.
} 
fréquemment avancé pour justifier le choix de l'écosystème préféré. Les visiteurs font le plus souvent abstraction des éléments perturbateurs pour vivre l'illusion aussi fortement que possible. Ainsi, interrogés sur l'aspect technique des écosystèmes, ils déclarent pour la plupart n'y avoir fait aucune attention: on n'est pas là pour voir des prouesses techniques. La minorité de visiteurs ayant étée sensible à ces installations déclare soit les avoir trouvées impressionnantes soit, comme le dit lui-même l'un d'entre eux, fort gênantes : " [c'est] dommage, on a l'impression d'être dans la réalité et quand on voit l'aspect technique on se souvient que c'est faux ». Le Biodôme satisfait ce besoin de rupture et de découverte d'un " Ailleurs » en proposant au visiteur un voyage sensoriel total. Il n'a pas l'impression de passer à l'extérieur d'une cage ou d'un milieu, aussi bien reconstitué soit-il : c'est le sentier qui se déroule au centre d'un milieu qui a une température, un degré d'humidité, une odeur propre. Par ailleurs, le fait que la représentation ne soit pas assignable à un endroit « réel » en particulier lui donne un caractère général qui accentue son pouvoir évocateur : puisqu'il ne s'agit pas d'un endroit particulier de la forêt tropicale, il s'agit de toute la forêt tropicale dans laquelle tout le monde peut projeter son imaginaire propre.

C'est ce projet même de représentation -c'est-à-dire de tentative de rendre présent ce qui est absent- du Biodôme, qui crée la complexité de son espace. Prenons l'exemple d'une partie du Saint-Laurent (figure 5). De fait, on peut, tout d'abord, noter la reproduction très réaliste du rocher, qui a été moulée sur un original existant effectivement dans le SaintLaurent marin. C'est cet espace qui nourrit l'illusion réaliste que produit le Biodôme. En tant que tel, il s'appuie sur une réalité matérielle qui existe bien dans le Biodôme (le rocher en ciment), mais il évoque une autre réalité matérielle et idéelle qui, elle, n'est pas au Biodôme : c'est le rocher qu'il représente, le paysage qui l'entoure et toutes les valeurs sociales qui y sont liées. En second lieu, on peut se tourner vers la fontaine qui prend sa base dans le rocher (en bas et au centre de l'image). Là encore la réalité matérielle est bien dans le Biodôme (l'appareil en acier inoxydable), mais cette fois la réalité matérielle et idéelle à laquelle il renvoie se situe dans le Biodôme. Il s'agit de l'institution publique qui est chargée de gérer la 


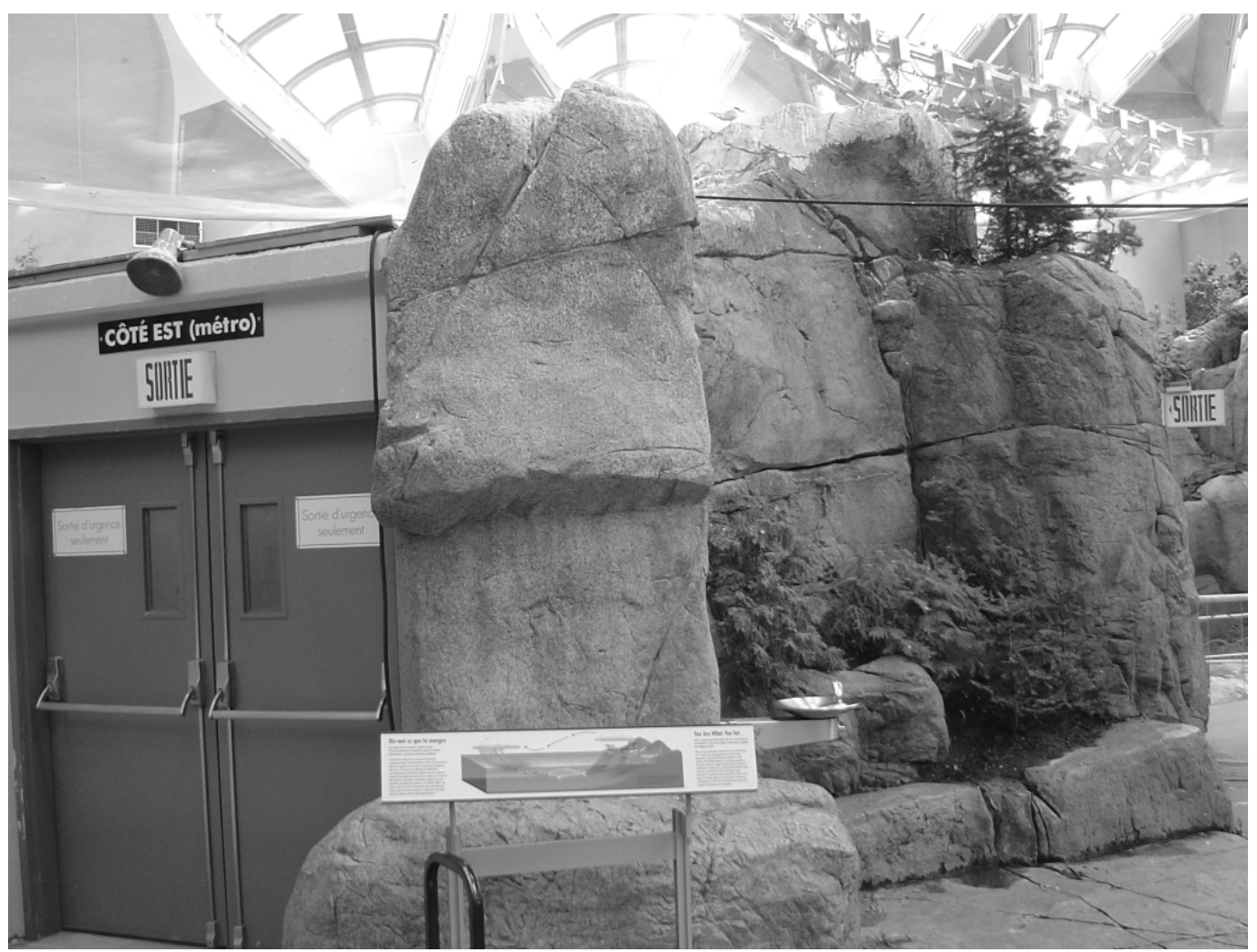

Figure 5 : Dans le Saint-Laurent marin, un espace hybride, 22/03/04, cliché de l'auteur

A hybrid space: the Saint-Lawrence seaway

collection d'animaux et de plantes et qui doit aussi s'occuper du confort des visiteurs, en leur offrant par exemple de l'eau à boire. C'est l'espace propre du Biodôme, qui menace de faire céder l'illusion réaliste que l'institution essaye de produire, en montrant que justement ce n'est qu'une représentation. Enfin, un troisième espace juxtaposé serait celui de la porte et du panneau "SORTIE Côté Est (métro) ». Là encore, l'espace matériel est celui du Biodôme, mais cette fois l'espace matériel et idéel de référence est celui de la ville, de l'extérieur du Biodôme, ce qui est finalement encore plus menaçant pour l'illusion réaliste mise en place, puisqu'elle remet le Biodôme dans son contexte montréalais, montrant bien que l'institution n'est qu'une petite pièce de « nature », dans une grande aire urbaine.

Le Biodôme présente donc un espace hybride, marqué par des tensions symboliques qui s'expriment tout au long du parcours par le même type de détails. L'institution, du fait de sa taille réduite et de ses limites nettes, semble pouvoir facilement être appréhendée comme un lieu, c'est-à-dire comme un espace dans lequel la distance n'est pas pertinente. D'un autre côté, son espace, loin de désactiver la distance, la suractive au contraire en faisant référence plus ou moins volontairement à une multitude d'autres contextes géographiques. En fait, le Biodôme joue justement sur la possibilité de présenter quatre milieux si lointains dans un espace si réduit, accentuant ainsi l'aspect exotique du micro-voyage. L'espace « réel» (le rocher en béton) et l'espace « imaginé » (la nature sauvage de l'Amazonie) se fondent ainsi dans une même réalité de l'espace représenté pour le visiteur, ce qui en produit justement la puissance.

\subsection{A la rencontre du sauvage et de l'exotique}

Si les gens viennent dans une institution comme le Biodôme, c'est avant tout pour se trouver face à face avec la nature dans ce qu'elle a de plus radical, donc de plus authentique. 
De fait, le public vient au zoo parce qu'il représente souvent dans un contexte urbain le seul moyen d'observer « en vrai » l'animalité sauvage par rapport au jardin public par exemple qui ne peut offrir qu'une nature jugée moins « sauvage », comme le remarque ce visiteur : "parce que, surtout dans une métropole, tu n'as pas l'occasion de voir des orignaux souvent ». En effet l'animal sauvage représente l'animal vrai parce que non dressé et donc libre de toute "déformation" humaine (au moins dans l'imagination du public). C'est que depuis le retournement de situation des années 1970 que nous avons décrit plus haut, la part animale de l'homme est très largement valorisée socialement, comme sa part de liberté et de spontanéité.

Ce sont bien les animaux qui rendent les écosystèmes intéressants pour les visiteurs : dans chaque milieu il existe une espèce vedette qui lui sert d'icône comme le castor ou les manchots. Le public cherche à entrer en interaction avec eux, en essayant de les toucher, en les pointant du doigt, en les nommant, en essayant d'obtenir leur attention en tapant sur la vitre ou en les appelant. D'autres encore s'essayent à des comportements d'imitation des cris ou des mimiques des animaux. Cela conduit les visiteurs à préférer les animaux actifs : les espaces où les animaux se cachent ou dorment sont ainsi très vite délaissés. Cette recherche de l'interaction et du contact traduit en fait une véritable volonté de confrontation avec l'animalité sauvage, ce que le premier projet de publicité du Biodôme mettait déjà en scène au printemps 1992. On nous présente ici une « expérience » dans laquelle l'homme et l'animal se situent à la même hauteur et se regardent droit dans les yeux dans les yeux, comme pour se jauger (figure 4).

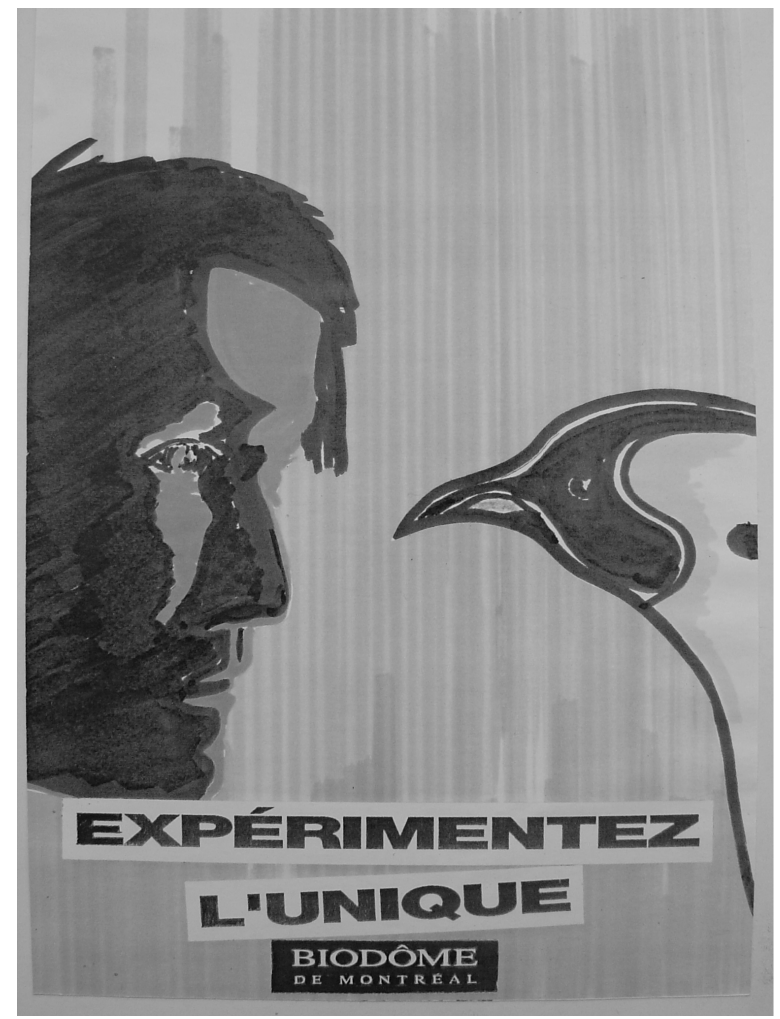

Figure 6 : Publicité pour le Biodôme, printemps 1992, cliché de l'auteur

An advertisement for the Biodôme

Cette nature sauvage se doit également d'être exotique. L'ailleurs exotique et sauvage devient le lieu de la quête de l'absolu, de vérité et de nouvelles opportunités. La forêt tropicale ou forêt vierge, dans l'imaginaire qu'elle suscite, est intouchée, libre de l'emprise humain, synonyme d'abondance. Le monde polaire, s'il ne présente pas ce côté paradisiaque, s'offre aussi comme un espace vierge et donc de découverte. Le danger de la confrontation 
avec de vastes espaces est conçu comme une épreuve d'où sort grandi l'homme victorieux, à la fois parce qu'il a réussi à dominer la sauvagerie de la nature, parce qu'il s'est renforcé en puisant des ressources au fond de lui-même afin de surmonter le péril et enfin parce qu'il a été plongé dans un monde « authentique ». Il est clair que les visiteurs ne pensent pas réellement se lancer dans une expédition mais ces idées constituent une sorte d'arrière-plan à leur visite qui les pousse à rechercher certains types de sensations. L'institution, consciente des ces besoins, propose d'ailleurs un Guide d'exploration du Biodôme de Montréal (Biodôme, 1993). Dans les faits, les visiteurs ne risquent évidement rien, mais c'est ce danger imaginaire lié à la confrontation avec la bête sauvage que le Biodôme doit réussir à mettre en scène à travers le camouflage de toutes les contentions et la création du sentiment d'immersion le plus fort possible.

\subsection{Construire un paysage national}

Si l'objectif affiché de l'institution est de présenter « certains des plus beaux milieux naturels ou écosystèmes des Amériques » (Biodôme de Montréal, 1993 , p.4), il apparaît clairement que plus de la moitié de la superficie totale est consacrée aux milieux québécois, ce qui correspond à une très large surreprésentation qui indique clairement sur quelle partie des Amériques l'attention est concentrée. Il est de fait assez étonnant a priori que le Biodôme ait dépensé de grosses sommes d'argent pour reconstituer et faire fonctionner dans un espace clos un écosystème qui se retrouve dans les environs assez immédiats de Montréal. La carte que présente le Biodôme, comme d'autres institutions du même type, fonctionne comme une sorte d'anamorphose qui traduit la vision du monde qu'ont ces concepteurs (Hancock et Staszak, 2002) : en l'occurrence ici, il s'agit bien d'une vision canadienne voire québécoise. De fait, peut-on alors parler de paysage national canadien alors que ne sont pas présents les paysages des grandes plaines de l'Est ou les forêts de la Colombie Britannique?

Il est singulier de voir que le Canada et le Québec ne sont perçu le plus souvent qu'à travers leurs caractéristiques naturelles : un endroit d'exploration et de découverte idéal, en somme. La wilderness est ainsi un élément majeur de définition de ce qu'est le Canada tant les visiteurs étrangers du Biodôme que pour les Canadiens eux-mêmes. L'importance de l'instrumentalisation de la nature dans le processus de constructions de la nature ont bien été étudiés pour les Etats-Unis d'Amérique (Opie, 1998), mais aussi le Canada (Bureau, 1984 ; Kaufmann et Zimmer, 1998). La constitution d'une histoire nationale a été chose délicate pour les jeunes nations d'Amérique du Nord, puisqu'elles manquaient tout simplement de lieux mémorables, d'églises, de cathédrales anciennes ou de tout autre monument qui puisse fonder avec suffisamment de légitimité une identité nationale. Ce sont les forêts, les montagnes, les chutes d'eau et les formes physiques surprenantes qui vont devenir les monuments nationaux. Les parcs nationaux vont donc avoir pour but de sanctuariser des espaces pour les préserver du changement et de les aménager afin de permettre au public de venir les admirer comme symbole de la nation (Opie, 1998). Au Canada en particulier, ce n'est qu'après la Confédération de 1867 que va peu à peu se dessiner la construction d'un sentiment national, tout d'abord à travers la Confederation School of Poets, en poésie puis surtout par le groupe des sept dans les années 1920. Ces artistes ont alors pour objectif de fonder une véritable peinture nationale afin de créer une communauté nationale comme ils le soutiennent euxmêmes dans leur première exposition majeure à Toronto en 1920. Ainsi, la figure 7 est un exemple représentatif de la manière dont commencent à se fixer les caractéristiques d'un paysage classique canadien : au premier plan une érablière ou des conifères sur un terrain légèrement vallonné, juste derrière un lac et enfin à l'arrière plan, une chaîne de montagne. 


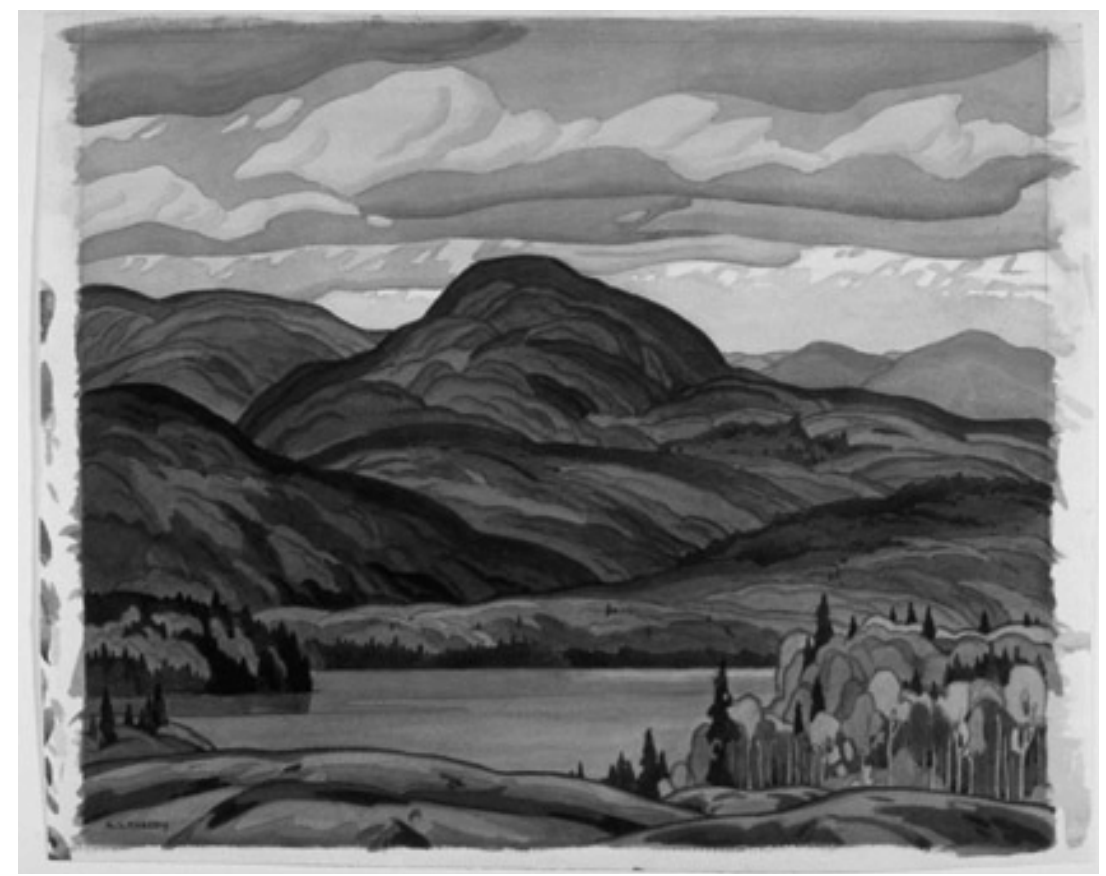

Figure 7 : Casson A. J., 1929, Pike Lake, McMichael Canadian Art Collection

Il faut voir que les travaux du groupe des sept a rencontré un succès notable parmi la nouvelle élite canadienne en formation mais également dans les couches populaires grâce aux nombreuses gravures qui étaient tirées à partir des originaux. Cette représentation du paysage canadien, loin d'être un modèle dépassé, est encore largement réactualisée aujourd'hui, notamment au travers des publicités diffusées dans le Canadian Geographic.

Le Biodôme reprend ce modèle paysager à travers le parcours-type tracé pour conduire au coeur des monuments nationaux, si on excepte évidement la forêt tropicale et en partie les pôles, plus liés à la question de l'exotisme. Les belvédères, qui offrent des points de vue remarquables sur les écosystèmes sont comparables par leur construction à ceux des parcs nationaux. Ils présentent les mêmes cascades (dans la forêt laurentienne), les mêmes escarpements rocheux (dans la foret laurentienne et le Saint-Laurent marin), les mêmes érablières tourmentées que ceux qu'on retrouve à travers la peinture, la littérature ou les parcs nationaux eux-mêmes. La forêt Laurentienne du Biodôme présente ainsi une version " parfaite » du lac canadien entouré d'une érablière et des traditionnels escarpements rocheux. Bien sur, il est impossible de recréer d'aussi vastes paysages qu'en extérieur mais l'ordonnancement en trois plans lac/forêt/montagne se retrouve bien. On ne tente pas de reproduire un « haut-lieu » (Debarbieux, 1995), facilement reconnaissable comme les chutes de Niagara. La question est plutôt de recréer un "paysage générique » (Meining et Jackson (ed.), 1979) qui n'existe pas à un endroit précis mais qui relève plutôt d'un modèle de paysage composé à partir du croisement de paysages réels, de représentations picturales ou textuelles et qui peut alors condenser en un lieu un idéal (ou un stéréotype ?) national qui est ici québécois.

\section{Le Biodôme ou l'illusion d'une nature libre}

\subsection{Une nature pour citadin}


Il est évident que le Biodôme ne nous présente qu'une version édulcorée de la nature. L'institution fonctionne, en effet, comme un service urbain, financé en tant que tel par la ville de Montréal et possédant d'ailleurs une clientèle essentiellement urbaine. Les visiteurs viennent faire une véritable promenade au Biodôme qui à l'avantage de présenter quelque chose de beaucoup plus sauvage et exotique qu'un simple jardin. C'est d'ailleurs un des arguments publicitaires du Biodôme avec des slogans comme «A Montréal, j'ai visité l'Antarctique » ou « En pleine ville, la mer». De fait, les visiteurs ne veulent pas retrouver les mêmes désagréments qu'en nature, à commencer par les efforts qu'il faut normalement y fournir. Comme l'indique un visiteur: «normalement la forêt tropicale c'est difficile d'avancer, ici il y a un chemin ». L'intérieur des écosystèmes a été conçu pour montrer le plus de choses possibles dans le moins d'espace possible, pour des raisons de place disponible, mais aussi pour éviter aux visiteurs à avoir trop à marcher. D'autre part, tout ce qui aurait pu être synonyme d'inconfort ou de gêne a été largement supprimé. Par exemple, le Biodôme fait particulièrement attention aux insectes ou aux odeurs qui ne sont pas acceptables. Enfin, le Biodôme ne peut se permettre de laisser ses différentes espèces se manger entre elles, comme ce serait le cas en nature, parce que ses collections deviendraient rapidement assez peu consistantes et aussi parce que les visiteurs urbains n'ont plus du tout l'habitude de voir des animaux être abattus ou mangés. En conséquence, le Biodôme a soigneusement sélectionné les espèces qu'on allait installer dans les écosystèmes et à mis en place des systèmes de contentions (comme les filets au-dessus de l'espace du lynx) qui visent à empêcher que ce genre d'évènements ne se produisent. Il est bien clair, au final, que le Biodôme se présente comme un espace contrôlé et strictement organisé par l'homme qui en a tout conçu.

En premier lieu, l'homme, en créant le Biodôme, s'est donné une sorte de rôle de démiurge. Ici, la tentative consistait bien à essayer de reproduire des écosystèmes dans leur totalité, c'est-à-dire, finalement la vie. On a donc une domination totale sur les animaux sauvages mais aussi sur des paramètres comme l'humidité ou la chute des feuilles dans la forêt Laurentienne qui dépendent entièrement des décisions humaines. Ainsi, on peut aussi voir dans le Biodôme une sorte de dernier avatar poussé à son extrême de la tradition occidentale de domination et de maîtrise de la nature.

En second lieu, le contrôle de l'homme s'exprime par l'organisation interne du Biodôme. En effet, classer les animaux, les plantes d'une certaine manière correspond à leur procurer un ordre et donc à les contrôler. Dès l'entrée, le visiteur reçoit un Guide de visite qui lui donne une vision d'ensemble du Biodôme en lui montrant une carte qui indique le parcours à suivre, les espèces qu'on y rencontre et dans quel ordre. Le sentier bétonné, aussi fermé et contraignant dans la liberté de mouvement soit-il, est très apprécié par les visiteurs, puisqu'il permet de ne rien oublier, de tout voir. Les visiteurs ont une attitude assez passive, ils attendent d'être guidé ou que les animateurs leur montrent les animaux qu'ils n'arrivent pas à voir. En somme, on pourrait dire que le Biodôme se présente comme une « machine à illusion », procurant des sensations « authentiques » à des citadins en mal de nature sauvage, tout en les laissant baigner dans l'univers de confort, de sécurité, d'urbanité en somme, auquel ils sont habitués.

\subsection{L'homme et la nature : des relations ambiguës}

Même si l'objectif affiché du Biodôme est bien de montrer que l'homme fait partie intégrante de la nature, il semble que sa réalisation matérielle est loin de produire ce résultat. En effet, contrairement au zoos classiques où plusieurs chemins s'ouvrent devant le visiteur, ici, le parcours est unique. Sur 500 mètres, le visiteur va suivre un sentier, en très grande partie bétonné, sauf dans certains endroits de la forêt Laurentienne et sur le belvédère du 
Saint-Laurent marin. Ce sentier est bordé des deux côtés par une barrière en verre qui arrive à mi-corps. De fait, le visiteur marche dans un espace différent de celui des écosystèmes, qui le sépare, tant matériellement que symboliquement, de la «nature » représentée. D'un côté, on trouve le sentier en béton -signe de technologie- où ne se trouvent que les hommes, puisque les animaux domestiques ne sont pas acceptés au Biodôme, et de l'autre se situent exclusivement les plantes et les animaux, puisque aucun homme n'est autorisé à y pénétrer en temps ordinaire. Dans ces conditions, il n'est pas très étonnant que le discours officiel du Biodôme sur l'appartenance de l'homme à la nature ne fonctionne pas. De fait, il est impossible d'instaurer une continuité entre l'homme et l'animal, il faudrait qu'il y ait des hommes dans l'écosystème, en représentation ou mieux en chair et en os. Voilà qui nous rappelle les «zoos humains » qui ont existé jusqu'en $1931^{4}$ et où étaient représentés des « sauvages » africains, le plus souvent, dans des enclos où ils se livraient à certaines activités (Bancel, 2002). Conserver un animal en captivité est encore acceptable dans certaines conditions, même si elle est contestée, par contre l'idée de maintenir des hommes en captivité pour la distraction ou même l'éducation d'autres hommes apparaît insensé aujourd'hui. Le projet du Biodôme est donc fondé sur une impossibilité absolue : pour pouvoir exister l'institution doit accepter d'aller à l'encontre de son idée de départ même en séparant totalement l'homme et la nature.

Keekok Lee (Lee, 1997) montre que, de fait, l'intérêt que la société porte à la nature est liée à la signification religieuse, culturelle, culinaire ou économique au point que souvent ce qui n'a aucune signification pour elle n'est même pas considéré comme naturel. Cette remarque est tout à fait exacte dans le cadre de l'imaginaire scientifique actuel de la conservation et du développement durable qui court désormais dans tous les débats concernant la nature et l'environnement, comme une sorte de dogme. Il est clair qu'au Biodôme cet aspect des choses est particulièrement souligné sous forme d'un discours stéréotypé. Ainsi, dans le Guide d'exploration du Biodôme de Montréal (Biodôme, 1993), on trouve des remarques comme " il faut sauver la forêt tropicale pluviale ! » ou « La ceinture 'verte' du globe rétrécit à un rythme alarmant... » (p. 31). Des panneaux sur la dégradation de la forêt tropicale ou des milieux marécageux complètent ce tableau noir qui semble décrire l'homme comme une sorte de destructeur.

Cependant, il est assez facile de voir que la nature est conceptualisée au Biodôme à partir du modèle scientifique désormais dominant, qui la voit avant tout comme une formidable banque de gênes et de ressources médicales ou autres. Ainsi, S. Whatmore et L. Thorne (Whatmore Sarah et Thorne Lorraine, 1998) ont montré que, finalement, le nom scientifique qu'on donne aux éléments de la nature aujourd'hui ne les conditionne pas moins que celui que les Romains donnait aux animaux voués au cirque. Pour les Romains, l'animal avait pour intérêt d'être un bon combattant ou d'être robuste, alors qu'aujourd'hui il s'agit de les objectiver selon un véritable marché de la gestion et de la protection du sauvage qui se fonde sur la diversité génétique et les ressources qu'il est possible d'en tirer. C'est bien ce courant que suit l'institution en indiquant par exemple que « cette diversité génétique est un précieux atout pour limiter les épidémies causées par des insectes ou des organismes microscopiques » Guide d'exploration du Biodôme de Montréal (Biodôme, 1993, p.32) L'idée de protéger les espèces en voie de disparition vient également de cette crainte de voir diminuer la diversité génétique avec les espèces. Les peurs liées à la pollution de l'environnement, à l'effet de serre pouvant amener des catastrophes ou à la disparition d'espèce, viennent directement de la question de la survie de l'homme. Ce qu'on cherche

\footnotetext{
${ }^{4}$ Lors de l'exposition coloniale de 1931, on avait en effet exhibé des Canaques, ce qui finalement entraîné une prise de conscience du public et la fin de ce genre de spectacles (Bancel, 2002).
} 
avant tout, c'est à protéger l'homme et assurer à l'espèce une existence aussi longue que possible.

\subsection{Un système d'ouverture et de fermeture au monde complexe}

La nature présentée est loin d'être « libre » parce qu'elle ne peut exister que dans des endroits bien précis : le Biodôme est le seul endroit à Montréal à mettre en scène de manière pérenne des animaux et une flore "sauvage» puisque cela constitue une certaine transgression de l'ordre urbain. Ainsi, le Biodôme fonctionne comme une sorte de bulle qui assure une séparation matérielle et symbolique avec le monde extérieur. L'architecture même du Biodôme, avec la grande voûte en béton qui fait de l'ensemble une sorte de coque, contribue à cette impression d'impénétrabilité. De fait, on ne peut pas voir ce qu'il se passe à l'intérieur de l'extérieur du bâtiment mais inversement il est impossible de voir l'extérieur, c'est-à-dire la ville de l'intérieur du Biodôme puisque la lumière est obtenue de manière zénithale par des lanterneaux. Le fait que les visiteurs viennent au Biodôme pour essayer de se laisser emporter par l'illusion qu'ils sont ailleurs, comme nous l'avons vu plus haut, contribue à la distinction : le visiteur vient se plonger dans un autre temps et un autre espace que celui de la ville environnante. Comme le Biodôme a pour fonction de recevoir des visiteurs, il doit être accessible. Pour ne pas briser la séparation qui existe entre les deux mondes, le Biodôme possède un véritable système de clapet qui régule les flux et permet ainsi une ouverture au monde contrôlée. Le visiteur doit, tout d'abord, passer la porte d'entrée avec des panneaux qui lui annoncent qu'il entre dans le Biodôme, puis il doit passer par les caisses et devant les gardes de sécurité, et enfin il doit passer des portes coulissantes qui lui donnent accès au premier écosystème. Ainsi, il n'y a pas moins de trois seuils différents qui permettent de contrôler qui entre, puisqu'il existe toute une série de règles à respecter, qui interdisent par exemple les animaux. Par ailleurs, on peut remarquer qu'il existe de véritables espaces de transition qui permettent de passer de manière moins brutale d'un espace à l'autre : il s'agit du hall d'entrée qui comporte les caisses, le restaurant et la boutique. De ce lieu, il est possible de voir le monde extérieur de la vitre à travers des baies vitrées mais il n'est pas encore possible de voir les écosystèmes qui sont derrière un autre seuil. Ainsi, le visiteur est déjà dans le Biodôme, sans voir ce qui en fait la raison d'être mais aussi la dangerosité, au moins symbolique : la nature sauvage.

\section{Conclusion}

Le Biodôme de Montréal est un révélateur assez efficace du «besoin de nature » ambigu qui presse une partie de la société occidentale : le public urbain est friand de la possibilité de rencontrer des espaces sauvages desquels il est ordinairement coupé. C'est qu'aujourd'hui, la nature est conçue comme quelque chose d'essentiellement bon et d'authentique, qui s'oppose à la ville et, de manière générale, à tout ce qui a trait à la culture humaine ou au progrès technologique. Ce qui fait la force du Biodôme est bien sa capacité à se présenter, malgré son aspect emminement technique et «artificiel», comme la reproduction impartiale d'une nature authentique. Or, il faut bien voir que le Biodôme propose une certaine vision du monde qui est loin d'être neutre. L'institution met en scène une nature édulcorée, entièrement au service de l'homme. De fait, il est certain que les visiteurs attendent de la visite qu'elle leur offre un frisson maîtrisé, loin des dangers et de la 
fatigue qu'impose un voyage «réel». Ainsi, le Biodôme est un lieu où la nature est totalement recrée et donc totalement contrôlée, depuis le degré d'humidité à la date de chute des feuilles, en passant par les espèces d'animaux qui sont présentes et la façon dont elles sont disposées. L'institution repose donc sur une sorte de projet démiurgique qui démontre, au final, la puissance et la supériorité humaine. De fait, il y a toujours une coupure radicale entre la nature et l'homme qui s'inscrit dans l'espace à plusieurs échelles, depuis la séparation matérielle du sentier bétonné central à celle plus profonde encore entre l'espace du Biodôme et celui de la ville, à travers un dispositif spatial qui assure une quasi-étanchéité physique et symbolique : la nature reste la marque de l'altérité radicale qui n'a pas droit de cité.

\author{
Ecole Normale Supérieure \\ 48 , bd. Jourdan \\ 75014 PARIS \\ jesteb@hotmail.fr
}

\begin{abstract}
Cet article est issu d'un travail de maîtrise conduit en 2003/2004, sous la direction de Jean-François Staszak (Paris I). Il a pour prolongement un mémoire de master 2 sur le jardin botanique de Naples, sous la codirection d'Emmanuel Jaurand (Paris XII) et de Jean-François Staszak (Paris I) et une thèse, en cours, sous la codirection de Christian Grataloup (Paris VII) et Bernard Debarbieux (Université de Genève) avec pour titre provisoire « Construire le monde : les jardins zoologiques comme miniature ».
\end{abstract}

\title{
Bibliographie
}

Anderson K., (2000), "'The beast within' : race, humanity and animality », Environment and Planning D : Society and Space, 18, pp. 301-320.

Batary É. et Hadouin-Fugier É., (1998), Zoos. Histoire des jardins zoologiques en Occident (XVIe-XXe siècles), Paris, La Découverte.

Biodôme de Montréal, (1993), Guide d'exploration du Biodôme de Montréal, Montréal, Biodôme.

Bancel N. et al. (dir.), (2002), Zoos humains. De la Vénus hottentote aux reality shows, Paris, La Découverte.

Bureau L., (1984), Entre l'Eden et l'Utopie, Montréal, Québec/Amériques.

Debarbieux B., (1995), "Le lieu, le territoire et trois figures de réthoriques », L'espace géographique, 24, 2, pp. 97-112

Estebanez J. (2006), Construire un ordre du monde : le Real Orto Botanico de Naples comme miniature, mémoire de master 2, Paris XII

Estebanez J. (2004), Entre «Eden et Utopie » : quelle nature pour le Biodôme de Montréal ?, mémoire de maîtrise, Paris I

Fait H. A. in Terrain, (2000), 34, « Les animaux pensent-ils ? », Paris, Gallimard.

Hancock C. et Staszak J.-F., (2002), « L'animal au zoo, enjeu de géographie politique. Le zoo de Mexico, de Moctezuma à l'écologie », Espaces et Sociétés, 110/111, p. 87-110.

Kaufmann E. et Zimmer O., (1998), "In search of the authentic nation: landscape and national identity in Canada and Switzerland », Nations and Nationalism, 4, 4, pp. 483-510.

Kisling V.N. (ed.), (2001), Zoo and Aquarium History. Ancient Animal Collections to Zoological Gardens, Boca Raton/London/New York/Washington, CRC Press.

Latour B., (1991), Nous n'avons jamais été modernes. Essai d'anthropologie symétrique, Paris, La Découverte. 
Latour B., (2001), L'espoir de Pandore, Pour une version réaliste de l'activité scientifique, Paris, La Découverte.

Lee K., (1997), « An animal: what is this? », Environmental values, 6, p. 393-410.

Meining D. W. et Jackson J.B. (ed.), (1979), The interpretation of ordinary landscape: geographical essay, New York, Oxford University press.

Moscovici S., (1968), Essai sur l'histoire humaine de la nature, Paris, Flammarion.

Opie J., (1998), Nature's nation: an environmental history of the United States, Fort Worth, Harcourt Brace College Publishers.

Philo C., (1995), "Animals, geography, and the city: notes on inclusions and exclusions », Environment and planning D: Society and space, 13, 6, pp. 655-681.

Quatre Temps, (1992), 16, 2, Montréal, Société des amis du jardin botanique.

Tarpin Ch. et Pitre R., (1996), «Exploration des écosystèmes au Biodôme de Montréal ; Evaluation sommative des comportements de visite, de l'efficacité communicationnelle des moyens d'exposition et de la rétention des messages ", Rapport final de l'étude commanditée par le Biodôme.

Whatmore S. et Thorne L., (1998), "Wild(er)ness: reconfiguring the geographies of wildlife », Transaction of the Institute of British Geographers, 23, p. 435-454.

Williams Raymond, (1997), Problems in materialism and culture, London/New York, Verso. Willis R. G. (ed.), (1990), Signifying Animals: Human meaning in the natural world, London/Boston, Unwin Hyman. 